\title{
Genetics Corner: Risk of Epilepsy in an Asymptomatic Infant with Prenatally Diagnosed Tuberous Sclerosis
}

Robin Dawn Clark, MD and Subhadra Ramaanthan, MSc, MS

\section{Case History:}

A healthy 10-month old male infant who had been prenatally diagnosed with tuberous sclerosis (TSC) was referred for a genetic consultation. The diagnosis of TSC in this child was made prenatally via gene testing on a chorionic villus sample by a maternalfetal medicine consultant. Fetal hydronephrosis was briefly noted on an ultrasound exam at $\sim 20$ weeks gestation, but there were no other signs of TSC in the fetus. The mother herself reported polycystic ovarian syndrome and chronic emesis but denied teratogenic exposures, diabetes, or other illnesses during her pregnancy. The baby was born at term by NSVD without complications and received routine newborn care. Birth weight and birth length were $7 \mathrm{lbs} 14 \mathrm{oz}$. and 20.5 inches, respectively. He passed his newborn hearing screen. He was discharged with his mother.

Since birth, this infant has been healthy and developing normally. He has not had any imaging studies or specialist evaluations to date. At the genetic clinic visit, his mother voiced concern about his risk for seizures after his upcoming MMR vaccination because his father, who also had TSC, had a seizure after his MMR vaccination as an infant.

The physical examination revealed a well-nourished, well-developed, alert, nondysmorphic male infant in no distress. He had one hypomelanotic macule on his right forearm. His neurologic examination was normal. He was socially engaged, vocal, and moved all extremities symmetrically. He demonstrated good muscle mass, strength, and tone.

\section{Developmental history:}

The patient met age-appropriate developmental milestones. He sat alone at 5-6 months of age and is almost standing unassisted at ten months. He said his first word at eight months of age and had a vocabulary of 3 words at ten months.

\section{Family and social history:}

The patient is the only child born to his parents. The patient's father, in his $30 \mathrm{~s}$, was diagnosed at age 30 with tuberous sclerosis as an incidental finding when he had a brain MRI for another indication. He has facial angiofibromas, migraine headaches, benign brain tumors, and a history of learning difficulties in school. He had a seizure with fever as an infant after receiving his MMR vaccine, but he has had no seizures since age 2 . A pathogenic variant in TSC1 was identified in the patient's father: c.2006_2007ins TTTAGGTTGCCTTT (p.Leu669Phefs*60).

The paternal grandmother and her mother are also affected by TSC. The paternal grandmother underwent genetic testing for tuberous sclerosis after the patient's father was diagnosed with TSC, and she has the same pathogenic variant. She has angiofibromas and brain and kidney tumors. She had "convulsions" when she was younger. The paternal great-grandmother had been clinically diagnosed with tuberous sclerosis in the past but had not had genetic testing. She has unspecified kidney issues.

\section{Assessment and counseling:}

This infant has a tuberous sclerosis complex (TSC) based on his family history, physical exam, and prenatal genetic test results. The hypomelanotic macule on his forearm is one of the earliest indicators of TSC and, in this patient, it is the sole manifestation of TSC at presentation. After an uneventful delivery, this infant was asymptomatic and went home with his mother without further interventions until ten months of age when he presented for an outpatient genetic consultation.

Although prenatal diagnosis provided the opportunity for early intervention in this case, the opportunity was missed, possibly because the fact that he was asymptomatic was overly reassuring to his family and his medical providers. Preventive management has an important role in the neonatal care plan of all affected newborns with TSC, even if asymptomatic. His mother's concern for seizure risk following routine immunizations motivated us to focus on the risk of epilepsy in TSC for this report and highlight preventive interventions appropriate at the time of diagnosis for all newborns with TSC.

\section{Background:}

TSC (OMIM 191100) is an autosomal dominant disorder caused by excessive activation of the mTOR signaling pathway by heterozygous inactivating pathogenic variants in either TSC1 or TSC2. It occurs in approximately 1 in 6000 births and is inherited from an affected parent in about one-third of cases. In the other approximately two-thirds of cases, the responsible gene variants occur as de novo events. The penetrance of TSC is thought to be $100 \%$, which means that all individuals with pathogenic variants express the disorder to varying degrees. However, the phenotype of TSC can be highly variable, even among affected members of the same family, so the disease does not "run true." Although no genotype-phenotype correlations regarding specific pathogenic variants in TSC1 have been reported, pathogenic variants in TSC1 are generally associated with a milder phenotype than pathogenic variants in TSC2.

In TSC, hamartomas develop in many organs: brain, heart, skin, kidneys, and lungs but the most severe manifestations are neurologic. The prevalence of intellectual disability is $40-70 \%$, and severe or profound disability is reported in $30-45 \%$ of patients. There is a strong association between mental handicap and epilepsy. Early-onset epilepsy, especially infantile spasms, is strongly linked to severe intellectual disability in TSC.

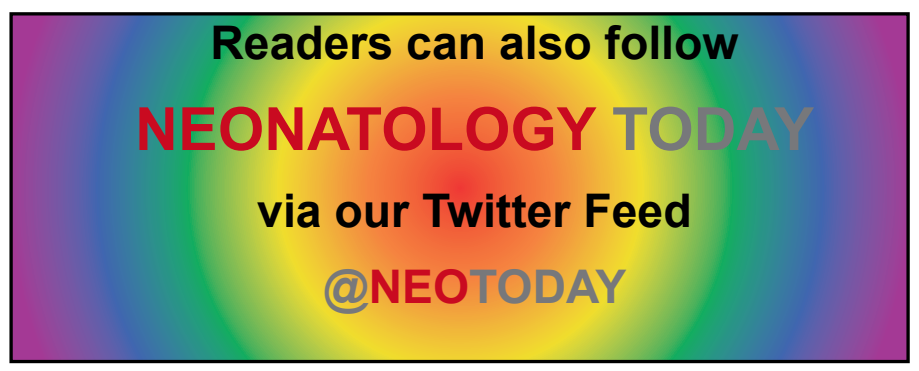




\section{Epilepsy and neurodevelopmental comorbidities}

Patients with TSC are at high risk of developing epilepsy. Seizures occur in about $80 \%$ of TSC infants, usually in the first year of life. Rapid treatment is important because seizures, especially uncontrolled seizures, increase the risk for TSC-associated neuropsychiatric disorders (TAND) such as intellectual disability/developmental delay and autism. Unfortunately, in approximately 65\% of those patients with TSC who develop epilepsy, seizures are severe and drug-resistant. Because mTOR activation can cause increased neuroexcitability and seizures, mTOR inhibitors have been studied for their antiepileptic therapeutic effect with positive results. The EXamining everolimus In a Study of Tuberous sclerosis 3 (EXIST-3) trial demonstrated decreased seizure frequency with the mTOR inhibitor everolimus in TSC-related refractory seizures in subjects aged 2-65 years old (French et al. 2016). Attention has now become focused on preventing epilepsy in at-risk infants with TSC by initiating treatment before clinical seizures are evident, and evidence is accumulating that this is beneficial.

"Vigabatrin is the most effective firstline monotherapy for TSC-associated infantile spasms and/or focal seizures in the first year of life (Curatolo et al., 2018)."

Vigabatrin is the most effective first-line monotherapy for TSC-associated infantile spasms and/or focal seizures in the first year of life (Curatolo et al., 2018). It has also been shown to be effective as a preventive therapy. In 2011 , Joswiak and colleagues demonstrated that vigabatrin, when initiated before the onset of seizures in at-risk infants with TSC, reduced the severity of their epilepsy and their risk of intellectual disability. In their study, a standard therapy group $(n=31)$ received antiepileptic treatment early but after the onset of seizures. A preventive therapy group $(n=14)$ was treated when active epileptic discharges were seen on EEG but before the onset of clinical seizures. At 24 months of age, the developmental delay was significantly more frequent and severe in the standard versus the preventive group ( $48 \%$ vs. $14 \% ; p=0.031$; mean IQ score 68.7 vs. 92.3; $p<0.05)$. The preventive group had a higher ratio of seizure-free children (93\% vs. $35 \%$; $p=0.004)$, a lower incidence of drug-resistant epilepsy ( $7 \%$ vs. $42 \%$; $p=0.021)$, and lower numbers who required polytherapy (21\% vs. $55 \%$; 0.039 ). These authors concluded, "preventative antiepileptic treatment of infants with tuberous sclerosis complex and high risk of epilepsy markedly improves their neurodevelopmental outcome and reduces the incidence of drug-resistant seizures."

Wu and colleagues (Wu et al., 2019) used serial routine 1 hour awake and sleep video EEGs as a biomarker for epileptogen- esis in at-risk infants with TSC. They studied 40 seizure-naïve infants who had TSC, aged seven months or younger, following them until two years of age with video EEGs at baseline, every six weeks until six months, every three months until 12 months, then every six months until 24 months of age. They found that interictal epileptiform discharges (IEDs) on EEG predicted clinical seizures by age 2 with a positive predictive value of $77.3 \%$ and a sensitivity of $85 \%$. Among the 32 infants who completed the study, 20 developed seizures, and 17 of these had persistent IEDs on multiple EEGs. In contrast, among the 12 infants who did not develop seizures, only 5 had IEDs on a single EEG that later resolved. The EEG changes preceded the onset of clinical seizures by a mean of 3.6 months. By demonstrating significant developmental decline only in the infants with ongoing seizures but not in infants who never developed seizures or whose seizures were controlled, these authors also showed that "decline in developmental outcome in infants with TSC is clearly linked to the persistence of seizures."

Most recently, the international EPISTOP clinical trial (Kotulska et al., 2020) compared the effectiveness of vigabatrin as a preventive therapy (after EEG evidence of epileptic activity) versus its use as conventional antiepileptic treatment (after the onset of clinical seizures) in TSC infants. Among 94 infants with TSC, aged four months or less, and without seizure history who participated in EEG surveillance, 54 were eligible for preventive treatment with vigabatrin in either a randomized arm or an open-label arm of the study. Of these, 25 had preventive treatment at the time of abnormal EEG, and 25 had no preventive treatment at the time of abnormal EEG. The median time from birth to the first clinical seizure was about four times longer with preventive treatment than conventional treatment (614 days vs. 124 days). Patients in the preventive treatment group were about three times more likely to remain free of clinical seizures over the study period ( $46 \%$ vs. $15 \%, p=0.011)$. The preventive treatment group had a lower median proportion of days with seizures $(17 \%$ vs. $62 \%, p=0.022)$ and less frequent drug-resistant epilepsy $(31 \%$ vs. $77 \%$, OR=0.15) than the conventional treatment group. None of the patients who received preventive treatment developed infantile spasms, whereas 10 of 25 patients on conventional therapy did. Importantly, there were no adverse events related to preventive treatment. The authors support the use of serial video-EEG monitoring "beginning at the time of diagnosis of TSC in infants and the immediate initiation of antiepileptic treatment with vigabatrin at the onset of epileptogenic activity."

We communicated this recommendation to the patient's pediatrician and family in the hope that an EEG could be performed prior to the patient's upcoming MMR vaccination. The patient was referred to the Pediatric Neurology service for brain MRI and further follow up.

The sum of current evidence is rapidly changing the standard of care for infants with TSC. Neonatal practitioners have an important role in advancing the health of these babies by ordering serial EEGs and advocating for preventive therapy, if needed, with

\begin{tabular}{|c|c|c|}
\hline $\begin{array}{l}\text { CONGENITAL } \\
\text { CARDIOLOGY }\end{array}$ & $\begin{array}{l}\text { The only worldwide monthly publication } \\
\text { exclusively serving Pediatric and Adult } \\
\text { Cardiologists that focus on Congenital/ } \\
\text { Structural Heart Disease (CHD), and } \\
\text { Cardiothoracic Surgeons. }\end{array}$ & $\begin{array}{c}\text { Subscribe Electronically } \\
\text { Free on the Home Page }\end{array}$ \\
\hline
\end{tabular}


vigabatrin when epileptogenic activity is present.

"The sum of current evidence is rapidly changing the standard of care for infants with TSC. Neonatal practitioners have an important role in advancing the health of these babies by ordering serial EEGs and advocating for preventive therapy, if needed, with vigabatrin when epileptogenic activity is present."

\section{Practical applications:}

1. Recognize the important role you have as a neonatal care provider in improving the lives of infants with tuberous sclerosis complex

2. Consult a pediatric neurologist whenever a newborn infant has TSC, even when the baby is asymptomatic, to initiate EEG surveillance, brain imaging and create a treatment plan

3. Anticipate that clinical seizures will develop in the first year of life in most infants with TSC.

4. Understand that early and close video EEG monitoring provide a biomarker for seizure susceptibility in TSC, and early treatment based on EEG monitoring significantly improves the neurodevelopmental outcome of young children with epilepsy.

5. Order EEG surveillance in infants with TSC prior to the onset of seizures to identify and treat early epileptiform changes

6. Recognize that antiepileptic treatment with vigabatrin begun prior to the onset of clinical seizures in infants with TSC improves neurologic outcome more than the same therapy initiated after the onset of seizures.

\section{References:}

1. Curatolo P, Nabbout $R$, Lagae L, et al. (2018). management of epilepsy associated with tuberous sclerosis complex: Updated clinical recommendations. Eur J Paediatr Neurol. 22(5):738-748. PMID: 29880258.

2. French JA, Lawson JA, Yapici Z, et al. (2016) Adjunctive everolimus therapy for treatment-resistant focal-onset seizures associated with tuberous sclerosis (EXIST-3): a phase 3, randomised, double-blind, placebo-controlled study. Lancet. 2016 Oct 29;388(10056):2153-2163. PMID: 27613521.

3. Jóźwiak S, Kotulska K, Domańska-Pakieła D, et al. (2011) Antiepileptic treatment before the onset of seizures reduces epilepsy severity and risk of mental retardation in infants with tuberous sclerosis complex. Eur J Paediatr Neurol. 2011 Sep;15(5):424-31. PMID: 21507691

4. Kotulska K, Kwiatkowski DJ, Curatolo P, et al., EPISTOP Investigators. (2020) Prevention of Epilepsy in Infants with Tuberous Sclerosis Complex in the EPISTOP Trial. Ann Neurol. 2020 Nov 12. doi: 10.1002/ana.25956. Epub ahead of print. PMID: 33180985.

5. Wu JY, Goyal M, Peters JM, et al. (2019). Scalp EEG spikes predict impending epilepsy in TSC infants: A longitudinal observational study. Epilepsia. 60(12):2428-2436. PMID: 31691264

Acknowledgment:

The authors would like to thank Dr. Stephen Ashwal for his helpful insights, scholarly input, and generous and always excellent advice.

The authors have no relevant disclosures.

NT
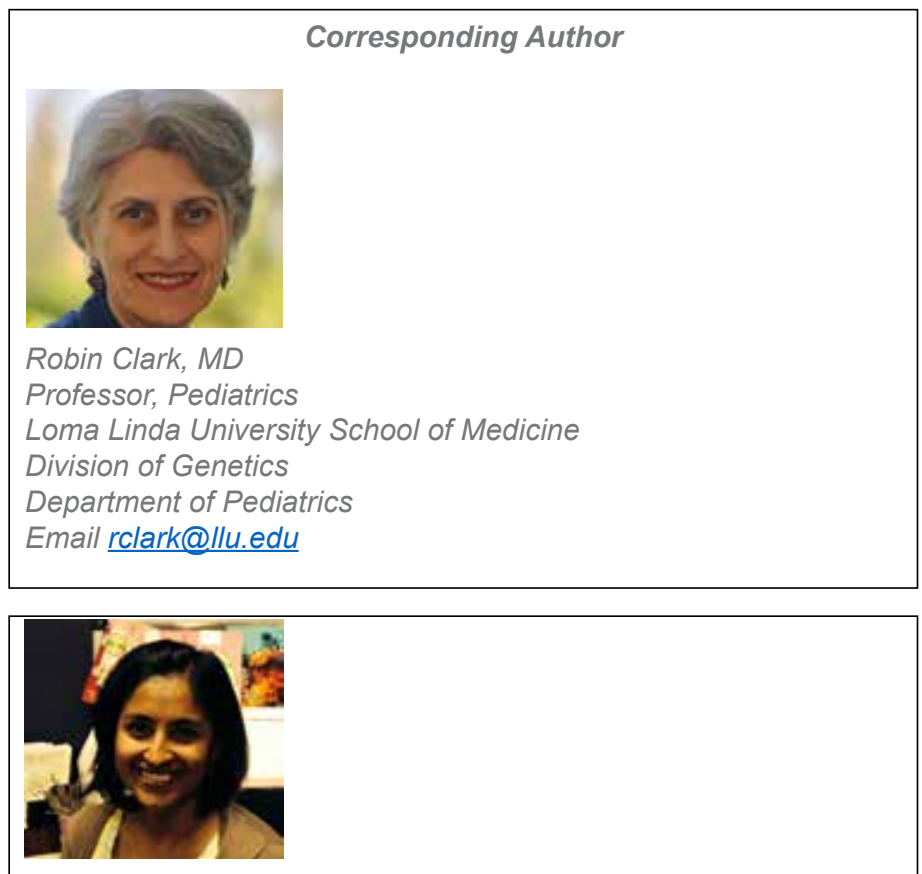

Subhadra (Subha) Ramanathan, M.Sc., M.S.

Licensed and Certified Genetic Counselor

Assistant Professor, Pediatrics

Loma Linda University Health

2195 Club Center Drive, Ste A

San Bernardino, CA 92408

SRamanathan@/lu.edu

NEONATOLOGY TODAY is interested in publishing manuscripts from Neonatologists, Fellows, NNPs and those involved in caring for neonates on case studies, research results, hospital news, meeting announcements, and other pertinent topics. Please submit your manuscript to: LomaLindaPublishingCompany@gmail.com 